\title{
Bigger is not always better: what the National Health and Hospitals Reform Commission report means for general practice
}

\author{
Michael R Kidd
}

T he final report of the Australian Government's National Health and Hospitals Reform Commission (NHHRC) was launched by Prime Minister Kevin Rudd on 27 July 2009. Much of its focus is on building on "the vital role of general practice", to strengthen primary health care as the "cornerstone of our future health system". ${ }^{1}$ The report has a welcome focus on equity, especially on shameful areas of past neglect, including the health of Aboriginal and Torres Strait Islander peoples, health in rural and remote areas, mental health, and dental health for all Australians. The report also reflects some changing social paradigms of the past decade, with a focus on patient-centred care, prevention, appropriate investment in patient-controlled e-health initiatives, and ensuring availability of high-quality health services to meet the growing demand from our ageing and increasingly informed population.

In his speech launching the report, the Prime Minister made a series of commitments, including:

- A commitment to primary care, because our GPs represent the front line of care.

- A commitment to sub-acute care to reduce preventable admissions to hospitals ..

- A commitment to investing in our health workforce and health infrastructure needs. ${ }^{2}$

As one of the seven guiding principles for reform, he identified "delivering comprehensive primary care or frontline care that properly connects GPs and community care on the one hand and acute and subacute care on the other". He stated that:

Our GPs in particular are under increasing pressure to provide services that are more appropriately provided by other health professionals, with the result that many patients either fall through the cracks or are forced into the hospital system. ${ }^{2}$

The Prime Minister also outlined three strategic options from the NHHRC report. Each option includes:

the Commonwealth taking responsibility for the following: all preventative care; all primary care; all hospital outpatients; all dental care; all aged care; all non-acute mental health services; 40 per cent of the funding for all acute hospital patients, and all sub-acute care delivered outside of our hospitals.

In itself, this would deliver the single most comprehensive health and hospital reforms since the introduction of Medicare.

Primary care services should be established as the cornerstone of a future person-centred health system, with the Commonwealth undertaking full funding responsibility

This should be supplemented by investment in local health infrastructure, so Australians have access to "one-stop-shop" community health services and a broad range of services with extended opening hours at more convenient times for patients. ${ }^{2}$

The government is now engaged in consultation to determine whether the federal government will assume full funding responsibility for all aspects of our health system. What ultimately happens in the hospital sector will have a significant impact on general practice and wider primary health care reforms.

\section{ABSTRACT}

- The National Health and Hospitals Reform Commission report has a focus on building on "the vital role of general practice", to strengthen primary health care as the "cornerstone of our future health system".

- The report proposes Comprehensive Primary Health Care Centres and Services that will deliver "one-stop" primary health care; but in health care, bigger is not always better.

- The biggest challenge for the reform process may well be bringing together the different cultures of the largely private primary health care services, funded by the federal government, and the public, primary and community health services, funded by the states and territories.

- The report pays little real attention to the reforms needed to address the social determinants of health.

- There is a clear need for action now on the report's most urgent recommendations for Aboriginal and Torres Strait Islander health, mental health, dental health and services for rural and remote communities.

- Diversity is a great strength of Australian general practice, and we must not lose it in the rush to reform.

MJA 2009; 191: 448-449

eMJA Rapid Online Publication 14 September 2009

The NHHRC report delivers a weighty set of concrete proposals for reform but is surprisingly light on detail and on evidence to support many of the recommendations affecting general practice. ${ }^{1}$ The report underplays the vital role that general practice has played and will continue to play. In contrast to the Prime Minister's speech, its wording is not particularly favourable towards general practice, preferring the terms "primary care" and "primary health care". The value of comprehensive, whole-person care delivered over time by general practice is not well acknowledged. The language of the report, with a focus on primary health care, risks disenfranchising many people working in general practice. The future roles of general practice and its dedicated health professional workforce remain opaque. Yet, strong primary health care in Australia has always relied on strong general practice.

The lack of specific focus in the report on the role of the GP in leading the primary care team is disappointing. It is hoped this will be further developed during the Prime Minister's ongoing consultation process. The evidence is clear that multidisciplinary planning of care to improve outcomes for people with chronic conditions needs to involve GPs; ${ }^{3,4}$ this is more specific than the recommendation in the report about enrolment with a "principal health care home". Any move to remove Australians' direct access to "my GP" will not be popular with the electorate.

The report seems focused on size, but in health care, bigger is not always better. Perhaps the optimum size for a primary health care centre is one where, in the words of Dr Chris Mitchell, President of the Royal Australian College of General Practitioners, the reception- 
ists know every patient by name (personal communication). Many people will prefer to continue attending smaller, more intimate general practices. And many private smaller general practices will prefer to continue specialising in offering services to specific populations. Diversity is one of the great strengths of Australian general practice, and we must not lose it in the rush to reform.

The report proposes Comprehensive Primary Health Care Centres and Services that will deliver "one stop" primary health care and will include both physical centres and virtual services. These appear to encompass the current networks of community-based services. The report seems fond of large community-based health care centres, but we need to heed the lessons from Australia's past large-scale investment in such structures - the outcomes of the Community Health Centres established during the Whitlam era, the recent challenges of creating the 31 GP Super Clinics, and the successful development of our nation's network of Aboriginal and Torres Strait Islander community-controlled health services. An important lesson is that any new primary health care infrastructure needs to work in partnership with local general practices and other primary care providers, rather than appear to compete.

The Australian Government plans to consider the NHHRC report for 6 months while consulting the public and health professionals. Yet, there is a clear need for action now on some of the report's recommendations. Whatever the outcome of consultations, there is an urgent need to improve Aboriginal and Torres Strait Islander health, mental health, dental health and services for rural and remote communities, and implementation should begin immediately. Although the focus on Aboriginal and Torres Strait Islander health is welcome, any initiatives need to build on the very strong and sound network of community-controlled health services already in existence across our country. It is hoped that the proposed National Aboriginal and Torres Strait Islander Health Authority will be controlled and staffed by Aboriginal and Torres Strait Islander people.

In addition, the focus of the report on Aboriginal and Torres Strait Islander people, people with serious mental illness and people living in remote and rural areas needs to be expanded to include all socially disadvantaged people. While proposing necessary improvement to health services, the report pays little real attention to the reforms needed to address the social determinants of health. This requires a whole-of-government approach.

If the government accepts responsibility for all primary health care and outpatient services, then mental health and the proposed "Denticare" and new child and family health services should all, of course, be included as integral parts of comprehensive primary health care.

The biggest challenge for the reform process may well be bringing together the different cultures of the largely private primary health care services, funded by the federal government, and the public, primary and community health services, funded by the states and territories. This will be an extraordinary exercise in change management. ${ }^{5}$ If the reforms are to work, then the two systems cannot work in parallel. They need to be integrated in a way that preserves the strengths of both systems and respects the commitment and passion of the health professionals working in both. Effective reform will need to explicitly recognise the perspectives of those involved in direct patient care. ${ }^{6}$

The replacement, or evolution — an important distinction - of Divisions of General Practice into Primary Health Care Organisations (PHCOs) has long been proposed, ${ }^{4}$ and indeed some Divi- sions have started to develop along these lines. However, the 250 000-500 000 population size to be served by a single PHCO may be appropriate for Australia's larger capital cities but may still be too large for many of the less densely populated parts of the country. Again, bigger may not always be better.

Proposals to expand the roles of nurse practitioners and other registered health professionals in areas of doctor shortage are an important step towards providing greater equity of access to care for many people living in remote locations. Training and support of any new practitioners will be essential, and care will be needed to ensure that the introduction of new practitioners does not lead to an exodus of those doctors who do work in remote locations.

The report has a strong focus on the training and continuing professional development of the health care workforce and the proposed establishment of a National Clinical Education and Training Agency. However, recommendations for investment in health service and clinical research are disappointingly thin.

It is easy to be critical. This report is a major milestone in Australian health care, and the commissioners and the Australian Government are to be congratulated. In our nation's history, opportunities for reform have been few and usually do not last long. Those of us who believe in the importance of general practice as the foundation of this nation's health care system need to make the most of this opportunity and ensure our opinion is heard during the consultation.

\section{Competing interests}

I chair the Australian Government's Ministerial Advisory Committee on Blood Borne Viruses and Sexually Transmissible Infections and am a member of the Australian Government's Medical Training Review Panel. I have served as chair or a member of past Australian Government committees, councils and boards. I am a past president of the Royal Australian College of General Practitioners and a current member of the executive of the Australian Health Care Reform Alliance.

\section{Author details}

Michael R Kidd, AM, MD, FRACGP, Executive Dean, ${ }^{1}$ and Honorary Professor $^{2}$

1 Faculty of Health Sciences, Flinders University, Adelaide, SA.

2 Faculty of Medicine, University of Sydney, Sydney, NSW.

Correspondence: michael.kidd@flinders.edu.au

\section{References}

1 National Health and Hospitals Reform Commission. A healthier future for all Australians: final report June 2009. http://www.nhhrc.org.au/internet/ nhhrc/publishing.nsf/Content/nhhrc-report (accessed Aug 2009).

2 Rudd K. Reforming Australian health care. Address at the John Curtin School of Medical Research, Australian National University, 27 July 2009 [transcript]. http://www.pm.gov.au/node/6069 (accessed Aug 2009).

3 Mitchell GK, Brown RM, Erikssen L, Tieman JJ. Multidisciplinary care planning in the primary care management of completed stroke: a systematic review. BMC Fam Pract 2008; 9: 44.

4 Harris M, Kidd M, Snowdon T. New models of primary and community care to meet the challenges of chronic disease prevention and management: a discussion paper for NHHRC, July 2008. http://www.health.gov. au/internet/nhhrc/publishing.nsf/ (accessed Aug 2009).

5 Kidd MR. What impact will the Australian Government's proposed national health care reforms have on Australian general practice [editorial]? Med J Aust 2009; 191: 55-57.

6 Degeling P, Kennedy J, Hill M. Mediating the cultural boundaries between medicine, nursing and management - the central challenge in hospital reform. Health Serv Manage Res 2001; 14: 36-48.

(Received 5 Aug 2009, accepted 24 Aug 2009) 